\title{
Measurement of the Lifetime Difference in the $B_{s}^{0}$ System
}

\section{Jason Kasper*i}

Boston University, USA

E-mail: jkasper@fnal.gov

\begin{abstract}
We present a study of the untagged decay $B_{s}^{0} \rightarrow J / \psi \phi$, using $450 \mathrm{pb}^{-1}$ of data collected in Run II of Fermilab experiment D $\varnothing$. In particular, we obtain an average lifetime difference in the $\left(B_{s}^{0}, \bar{B}_{s}^{0}\right)$ system of $1.39_{-0.16}^{+0.13}(\text { stat })_{-0.02}^{+0.01}(s y s t) p s$, a relative width difference of the mass eigenstates, $\Delta \Gamma / \bar{\Gamma} \equiv\left(\Gamma_{L}-\Gamma_{H}\right) / \bar{\Gamma}$, of $0.25_{-0.38}^{+0.28}$ (stat) ${ }_{-0.04}^{+0.03}$ (syst), and the CP-odd fraction at time zero, $R_{\perp}=0.16 \pm 0.10$ (stat) \pm 0.02 (syst) .
\end{abstract}

International Europhysics Conference on High Energy Physics

July 21st - 27th 2005

Lisboa, Portugal

*Speaker.

$\dagger^{\dagger} \varnothing$ Collaboration 
The reaction in this study is the hadronic decay, $B_{s}^{0} \rightarrow J / \psi \phi$, via the subprocess, $b \rightarrow \bar{c} c s$. Only $J / \psi$ decays to $\mu^{+} \mu^{-}$and $\phi$ decays to $K^{+} K^{-}$are considered. Note that the decay for the conjugate state, $\bar{B}_{s}^{0}$, is identical. This gives an opportunity to determine the lifetime difference in the $\left(B_{s}^{0}, \bar{B}_{s}^{0}\right)$ system in an untagged sample by separating the $C P$ eigenstates via angular distribution and fitting for each state's lifetime. Note that untagged here refers to the lack of knowledge, at its decay, as to whether the particle is the $B_{s}^{0}$ or $\bar{B}_{s}^{0}$. The data presented here reflect a recent DØ Collaboration published article, a more in depth discussion of this analysis can be found there [1].

In the standard model, mixing occurs such that there will be a significant lifetime difference between the mass eigenstates of the $\left(B_{s}^{0}, \bar{B}_{s}^{0}\right)$ system. The light and heavy mass eigenstates are defined as, $\left|B_{s}^{L}>=p\right| B_{s}^{0}>+q \mid \bar{B}_{s}^{0}>$ and $\left|B_{s}^{H}>=p\right| B_{s}^{0}>-q \mid \bar{B}_{s}^{0}>$ with $|p|^{2}+|q|^{2}=1$. If we define their widths as $\Gamma_{H}$ and $\Gamma_{L}$, respectively, the standard model predicts, $\Delta \Gamma / \bar{\Gamma} \approx(12 \pm 5) \%$, where $\Delta \Gamma \equiv \Gamma_{L}-\Gamma_{H}$ and $\bar{\Gamma} \equiv\left(\Gamma_{H}+\Gamma_{L}\right) / 2$. In the standard model, the mass and $C P$ states parameters are approximately equal (they are related by a rotation, $\phi$, where $\phi \approx-0.03$ ). In the limit of no $C P$ violation, the two would be identical. Physics beyond the standard model can also be probed by looking for larger $\phi$ which would appear as a reduction in $\Delta \Gamma / \bar{\Gamma}[2]$. For the results below, $\phi$ is considered negligible.

The data was collected in the DØ detector, between June 2002 and August 2004, and represents $450 \mathrm{pb}^{-1}$. DØ has quite pure and efficient muon detection which is crucial for this analysis [3]. Muon coverage is large, extending to absolute pseudorapidity less than two. The muons from the $J / \psi$ are both required to be matched to a central track and a low $p_{T}$ cut is used $\left(p_{T}>2-4 \mathrm{GeV}\right.$, depending on location in the detector). Two oppositely charged muons are fit to a common vertex to define a $J / \psi$. The $J / \psi$ mass resolution is approximately $70 \mathrm{MeV}$. A dimuon trigger was employed, but no impact parameter cuts were allowed. Kaons are given a $p_{T}$ cut of $0.7 \mathrm{GeV}$ and are fit to a common vertex to define a $\phi$. If the $J / \psi$ and $\phi$ are consistent with coming from the same vertex (but with distinct tracks), and they fall into the mass windows $(2.90-3.25) \mathrm{GeV}$ and $(1.01-1.03) \mathrm{GeV}$, respectively, a $B_{s}^{0}$ candidate is declared (except where noted, the term $B_{s}^{0}$ implies also the conjugate state). There are 9699 candidate events. Figure 1 shows the invariant mass distribution for these

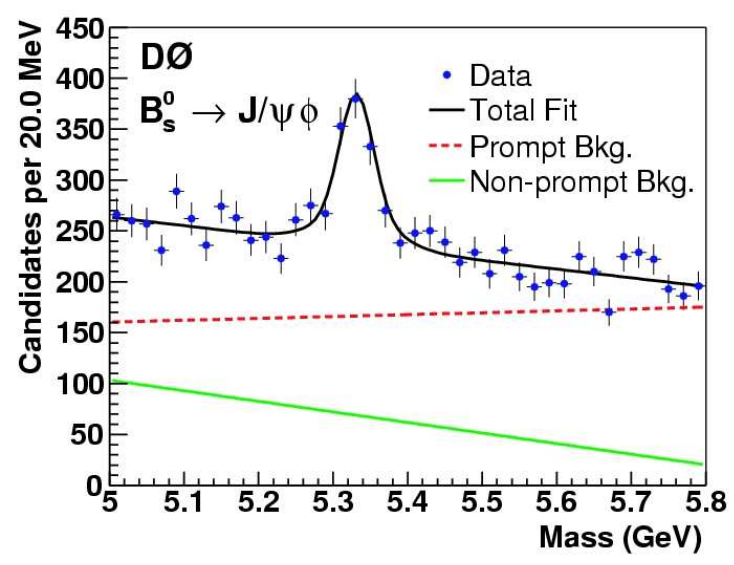


candidates, where the fit is the result of the maximum likelihood described below.

The $B_{s}^{0} \rightarrow J / \psi \phi$ decay can be parameterized by three angles; the azimuthal $(\xi)$ and polar $(\theta)$ angles of the $\mu^{+}$with respect to the proton beam direction in the $J / \psi$ rest frame, and the polar angle $(\zeta)$ of the $K^{+}$with respect to the $J / \psi$ direction in the $\phi$ rest frame. The transversity is defined as $\cos \theta$ and is particularly sensitive to the $\mathrm{CP}$ quantum state of the reaction. We find it useful to integrate the differential cross section over the non-transversity angles to obtain:

$$
\frac{d^{2} \Gamma}{d \cos \theta d t} \propto\left[N\left(\left|A_{0}(0)\right|^{2}-\left|A_{\|}(0)\right|^{2}\right) e^{-\Gamma_{L} t}\left(1+\cos ^{2} \theta\right)+2\left|A_{\perp}(0)\right|^{2} e^{-\Gamma_{H} t} \sin ^{2} \theta\right]
$$

where the proportinality factor, $N$, is very small and the $A$ 's represent the linear polarization amplitudes for the various CP eigenstates. Note that we do not use all three angles for our fits and so do not determine all amplitudes. The CDF result is used for the $C P$ even contribution, $\left|A_{0}(0)\right|^{2}-\left|A_{\|}(0)\right|^{2}=0.355 \pm 0.066[4]$.

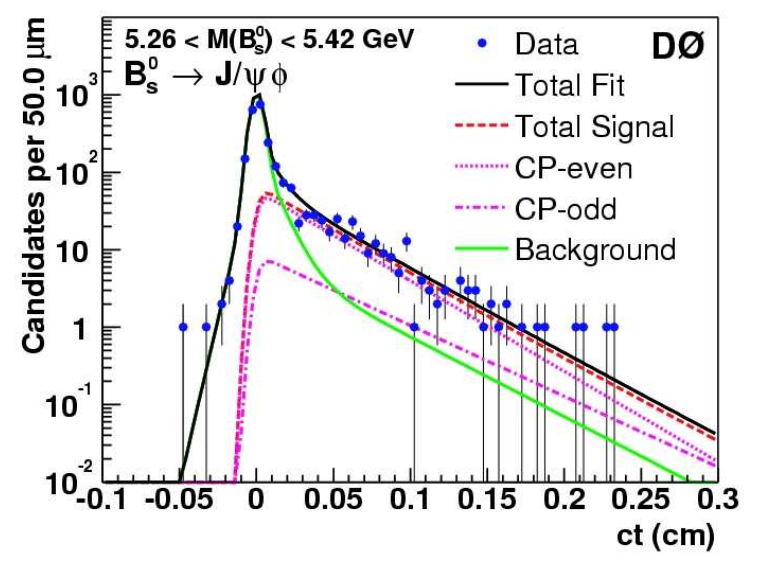

(a)

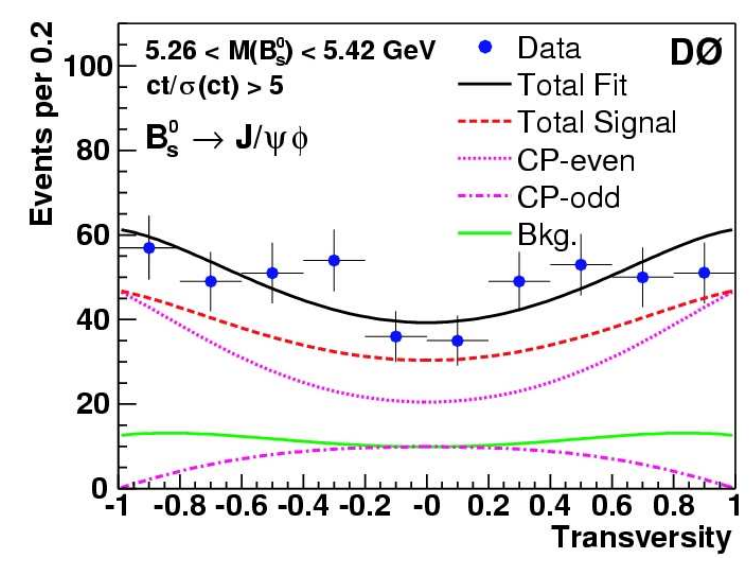

(b)

Figure 2: Proper decay length, $c t$, of $B_{s}^{0}$ candidates in the signal mass region (a). Cosine of the transversity in the signal mass region (b); this distribution is not from the fi t a ct cut is made to show the distribution for non-prompt $J / \psi$.

The data is fit using an unbinned maximum likelihood simultaneously fitting for the mass, lifetime, and transversity. There are a total of 19 free parameters. The mass is fit with a double gaussian with a common mean in the signal region and two first order polynomials (for the prompt and non-prompt backgrounds) outside the signal region. The lifetime is fit with 2 exponentials multplied with two gaussians (for CP odd and even components), one gaussian (for the prompt background), and three exponentials (one for the positive and two for the negative non-prompt backgrounds). Finally, the transversity is fit with polynomials of the form $G(\theta)\left(1+\cos ^{2} \theta\right)$ for $C P$ even $F(A)\left(1-\operatorname{coc}^{2} A\right)$ for $C P$ add and $G(A)$ senarately for nromnt and non-nrnmnt hackornunds 
cut to emphasize the non-prompt contribution) are shown in figure 2. For a discussion on how we measure lifetime, see reference [1].

The fit gives the following results: average lifetime of the mass eignestates, $c \bar{\tau}=416_{-48}^{+39} \mu \mathrm{m}$, $C P$ odd fraction at time zero, $R_{\perp}=0.16 \pm 0.10$, and the relative fractional width, $\Delta \Gamma / \bar{\Gamma}=0.24_{-0.38}^{+0.28}$. In figure 3 we present the $1 \sigma$ contour from the fit with $c \bar{\tau}$ plotted against $\Delta \Gamma / \bar{\Gamma}$. The DØ result is shown standalone and when constrained by the world average as obtained from the flavor specific values (from semi-leptonic $B_{s}^{0}$ decays). D $\emptyset$ in both cases is consistent with the standard model. Note that the CDF result is consistent with DØ but at the $1 \sigma$ level, not with the standard model. The flavor specic constraint gives, for $\mathrm{D} \emptyset, \Delta \Gamma / \bar{\Gamma}=0.25_{-0.15}^{+0.14}$ and $\bar{\tau}=1.39 \pm 0.06 \mathrm{ps}$. The other plot in the figure shows the result from the Heavy Flavor Averaging Group (the "direct" measurements are dominated by $\mathrm{D} \emptyset$ and $\mathrm{CDF}$ ). Note that the result is $3 \sigma$ from 0 and about $1 \sigma$ from the standard model prediction. Finally, with the calculation of the average lifetime in the $B_{d}^{0}$ system [1], we get a ratio, $\bar{\tau}_{B_{s}^{0}} / \bar{\tau}_{B_{d}^{0}}=0.91 \pm 0.09$ (stat) \pm 0.003 (syst) ps; in good agreement with theory which predicts that the average lifetime in the $B$ meson systems should be independent of light quark flavor.

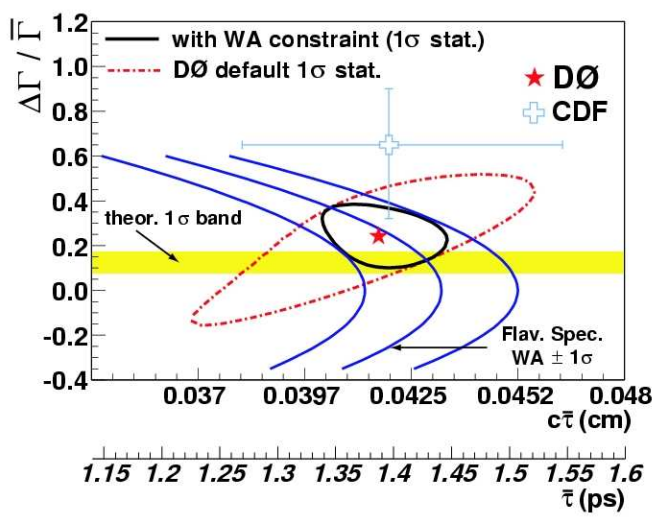

(a)

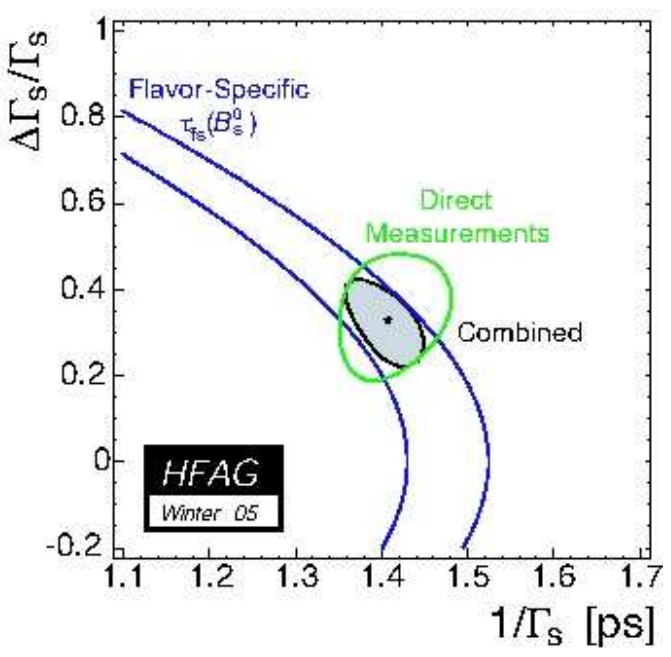

(b)

Figure 3: Statistical $1 \sigma$ contour for $\mathrm{D} \varnothing$ alone and with constraints from the world average fhvor specific channels (a). HFAG $1 \sigma$ contour with D $\varnothing$ results included (b). Note that (b) uses different axes notation, but they are the same meanings as in (a).

\section{References}

[1] DØ Collaboration, V. Abazov, et al., "Measurement of the Lifetime Difference in the $B_{s}^{0}$ System", Phys. Rev. Lett., 95171801 (2005)

[2] I. Dunietz, R. Fleischer, and U. Nierste, Phys. Rev. D63 114015 (2001)

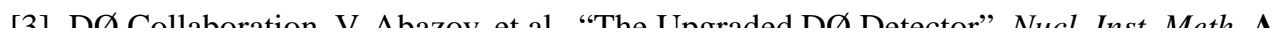

